\title{
Topological Self-Assembly of Highly Symmetric Lanthanide Clusters: A Magnetic Study of Exchange- Coupling "Fingerprints" in Giant Gadolinium(III) Cages
} DOI:

10.1021/jacs.7b09996

\section{Document Version}

Accepted author manuscript

Link to publication record in Manchester Research Explorer

Citation for published version (APA):

Qin, L., Zhou, G-J., Yu, Y-Z., Nojiri, H., Winpenny, R., \& Zheng, Y-Z. (2017). Topological Self-Assembly of Highly Symmetric Lanthanide Clusters: A Magnetic Study of Exchange-Coupling "Fingerprints" in Giant Gadolinium(III) Cages. Journal of the American Chemical Society. https://doi.org/10.1021/jacs.7b09996

\section{Published in:}

Journal of the American Chemical Society

\section{Citing this paper}

Please note that where the full-text provided on Manchester Research Explorer is the Author Accepted Manuscript or Proof version this may differ from the final Published version. If citing, it is advised that you check and use the publisher's definitive version.

\section{General rights}

Copyright and moral rights for the publications made accessible in the Research Explorer are retained by the authors and/or other copyright owners and it is a condition of accessing publications that users recognise and abide by the legal requirements associated with these rights.

\section{Takedown policy}

If you believe that this document breaches copyright please refer to the University of Manchester's Takedown Procedures [http://man.ac.uk/04Y6Bo] or contact uml.scholarlycommunications@manchester.ac.uk providing relevant details, so we can investigate your claim.

\section{OPEN ACCESS}




\section{Article}

\section{Topological Self-Assembly of Highly-Symmetric Lanthanide Clusters: A Magnetic Study of Exchange- Coupling "Fingerprints" in Giant Gadolinium(III) Cages}

Lei Qin, guojun zhou, You-Zhu Yu, Hiroyuki Nojiri, Christian

Schröder, Richard E. P. Winpenny, and Yan-Zhen Zheng

J. Am. Chem. Soc., Just Accepted Manuscript • DOI: 10.1021/jacs.7b09996 • Publication Date (Web): 16 Oct 2017

Downloaded from http://pubs.acs.org on October 26, 2017

\section{Just Accepted}

"Just Accepted" manuscripts have been peer-reviewed and accepted for publication. They are posted online prior to technical editing, formatting for publication and author proofing. The American Chemical Society provides "Just Accepted" as a free service to the research community to expedite the dissemination of scientific material as soon as possible after acceptance. "Just Accepted" manuscripts appear in full in PDF format accompanied by an HTML abstract. "Just Accepted" manuscripts have been fully peer reviewed, but should not be considered the official version of record. They are accessible to all readers and citable by the Digital Object Identifier (DOI®). "Just Accepted" is an optional service offered to authors. Therefore, the "Just Accepted" Web site may not include all articles that will be published in the journal. After a manuscript is technically edited and formatted, it will be removed from the "Just Accepted" Web site and published as an ASAP article. Note that technical editing may introduce minor changes to the manuscript text and/or graphics which could affect content, and all legal disclaimers and ethical guidelines that apply to the journal pertain. ACS cannot be held responsible for errors or consequences arising from the use of information contained in these "Just Accepted" manuscripts.

\section{ACS Publications}




\title{
Topological Self-Assembly of Highly-Symmetric Lanthanide Clusters: A Magnetic Study of Exchange-Coupling "Fingerprints" in Giant Gadolinium(III) Cages
}

\author{
Lei Qin, ${ }^{1, \|}$ Guo-Jun Zhou, ${ }^{1, \|}$ You-Zhu Yu, ${ }^{1, \$}$ Hiroyuki Nojiri, ${ }^{2}$ Christian Schröder, ${ }^{3,{ }^{*}}$ Richard E. P. \\ Winpenny ${ }^{4, *}$ and Yan-Zhen Zheng ${ }^{1, *}$ \\ ${ }^{1}$ Frontier Institute of Science and Technology (FIST), Xi'an Jiaotong University, Xi'an 710054, P. R. China. \\ 2 IMR, Tohoku University, Tohoku, Japan. \\ ${ }^{3}$ Bielefeld Institute for Applied Materials Research, Bielefeld University of Applied Sciences, D-33619 Bielefeld, Ger- \\ many. \\ ${ }^{4}$ School of Chemistry, The University of Manchester, Oxford Road, Manchester, M13 9PL, United Kingdom. \\ KEYWORDS: High-symmetry •Antiferromagnetic $・$ Heisenberg model $\bullet$ Gadolinium $\bullet$ Polyhedra
}

\begin{abstract}
The creation of a perfect hollow nanoscopic sphere of metal centres is clearly an unrealisable synthetic challenge. It is however an inspirational challenge, from the viewpoint of chemical architecture and also as finite molecular species may provide unique microscopic insight into the origin and onset of phenomena such as topological spin-frustration effects found in infinite 2D and 3D systems. Herein, we report a series of high symmetry gadolinium(III) $(S=7 / 2)$ polyhedra, $\mathrm{Gd}_{20}, \mathrm{Gd}_{32}, \mathrm{Gd}_{50}$ and $\mathrm{Gd}_{60}$, to test an approach based on assembling polymetallic fragments that contain different polygons. Structural analysis reveals the $\mathrm{Gd}_{20}$ cage resembles a dodecahedron; the vertices of the $\mathrm{Gd}_{32}$ polyhedron exactly reveal symmetry $\mathrm{O}_{\mathrm{h}}$; $\mathrm{Gd}_{50}$ displays an unprecedented polyhedron in which an icosidodecahedron $\mathrm{Gd}_{30}$ core is encapsulated by an outer $\mathrm{Gd}_{20}$ dodecahedral shell with approximate $\mathrm{I}_{\mathrm{h}}$ symmetry; and the $\mathrm{Gd}_{60}$ shows a truncated octahedron geometry. Experimental and theoretical magnetic studies show that this series produces the expected antiferromagnetic interaction that can be modelled based on classical spins at the Gd sites. From the magnetization analyses we can roughly correlate the derivative bands to the Gd-O-Gd angles. Such a magneto-structural correlation may be used as "fingerprints" to identify these cages.
\end{abstract}

\section{INTRODUCTION}

One of the attractions of $\mathrm{C}_{60}$ as a molecule is how closely it approaches a sphere. It is not a perfect sphere, as that is a chemical impossibility, but approaches a sphere by having a mixture of pentagonal and hexagonal faces. ${ }^{1}$ If we compare the five Platonic solids with Archimedean solids we immediately see that in the majority of cases an Archimedean solid, where the faces are inequivalent regular polygons, are far closer to a sphere, than the Platonic solids where all faces are identical (Figure 1). ${ }^{2}$ Therefore we reasoned that to construct a metal polyhedron that approaches a sphere in structure, a mixture of different polygons should be used. ${ }^{3}$ This may initially appear counterintuitive. In some ways it builds from previous topological analysis which showed that a "magic" pentagon $\left\{(\mathrm{Mo}) \mathrm{Mos}_{5}\right\}$ is critical in constructing highly-symmetric spherical polyoxometallates (POMs): ${ }^{4}$ implicit in that analysis is that some of the polygons linking the magic pentagons have a different number of edges.

Highly symmetric arrangements of paramagnetic centres are expected to show exotic spin frustration effects if they are coupled antiferromagnetically as it is impossible to align all spins anti-parallel to all nearest neighbours. ${ }^{5}$ This can lead to unusual fundamental new physics such as metamagnetic phase transitions and unexpected hysteresis effects. ${ }^{6}$ However, a necessary ingredient in order to find such effects experimentally requires spin centres that behave like pure spins, i.e. any significant single ion anisotropy is absent.

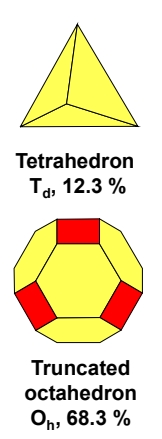

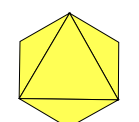

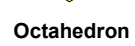
$\mathrm{O}_{\mathrm{h}}, \mathbf{3 1 . 8} \%$
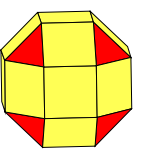

Rhombicuboctahedro $\mathrm{O}_{\mathrm{h}}, \mathbf{7 6 . 0} \%$

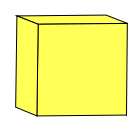

Cube
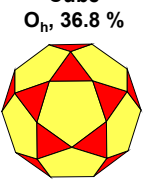

Icosidodecahedron $\mathrm{I}_{\mathrm{h}}, \mathbf{7 8 . 0} \%$

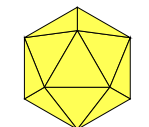

Icosahedron Dodecahedron $I_{h}, 60.5 \% \quad I_{h}, 66.5 \%$
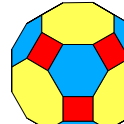

Truncated cuboctahedron $\mathrm{O}_{\mathrm{h}}, \mathbf{8 0 . 2} \%$

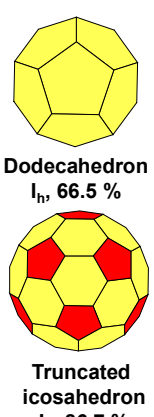

$I_{h}, 86.7 \%$
Figure 1. All Platonic (first row) and selected Archimedean (second row) polyhedral solids, together with the point group and volume ratio ( $V_{\text {polyhedron }} / V_{\text {circumsphere). The higher the ratio }}$ the closer the polyhedron is to the circumsphere.

Here we build high symmetry gadolinium(III) cages (Table S1), using a topological knowledge of lanthanide (Ln) fragments, such as the $\left\{\operatorname{Ln}_{3}\left(\mu_{3}-\mathrm{OH}\right)\right\}$ triangle, $\left\{\operatorname{Ln}_{4}\left(\mu_{4}-\right.\right.$ $\mathrm{OH})\}$ square, $\left\{\mathrm{Ln}_{4}\left(\mu_{4}-\mathrm{OH}\right)_{4}\right\}$ tetrahedron, $\left\{\operatorname{Ln}_{5}\left(\mu_{5}-\mathrm{Cl}\right)\right\}$ pentagon and $\left\{\operatorname{Ln}_{6}\left(\mu_{6}-\mathrm{CO}_{3}\right)\right\}$ hexagon (Figure S1). Gadolinium(III) was chosen as its spin moment is very large $(S=$ $7 / 2$ ) and its orbital moment is zero and hence it is ex- 
pected to behave like a classical spin centre. This allows us to model such systems classically since a quantum treatment is impossible due to the large number of interacting magnetic moments.

\section{RESULTS}

Synthetic strategy and overview of structural motifs. Our synthetic strategy involves using simple anions as weak templates in the presence of other multidentate ligands; on occasion trapping a polymetallic intermediate allows us to build pseudo-spherical cages that are not otherwise accessible.

For example: a $\left\{\mathrm{Gd}_{60}\right\}$ cage of formula $\left[\mathrm{Gd}_{60}\left(\mathrm{CO}_{3}\right)_{8}(\mathrm{OH})_{96}(\mathrm{dmp})_{28}\left(\mathrm{NO}_{3}\right)_{8}\left(\mathrm{H}_{2} \mathrm{O}\right)_{8}\right]\left(\mathrm{NO}_{3}\right)_{32} \quad \mathbf{G d}_{60}$ can be made directly from reaction of hydrated gadolinium nitrate with 2, 2-dimethylol propionic acid (Hdmp) at $130{ }^{\circ} \mathrm{C}$ in mixed solvents $\left(\mathrm{CH}_{3} \mathrm{CN} / \mathrm{CH}_{3} \mathrm{OH} / \mathrm{H}_{2} \mathrm{O}\right)$ under sol- vothermal conditions (see Supporting information for details). The aqueous solution involved contains a large quantity of base and is exposed to air prior to reaction, which leads to formation of carbonate which is the simple anion that favours the formation of $\mathbf{G d}_{\mathbf{6 0}}$ (Figures 2, 4 and S2). A similar $\left\{\mathrm{Er}_{60}\right\}$ cage has been reported by Zheng et al. ${ }^{7}$ The structure can be viewed as a truncated octahedron of Gd(III) tetrahedron units with 6.222-bridging (Harris notation $\left.^{8}\right) \mathrm{CO}_{3}{ }^{2-}$ anions on the slightly distorted hexagonal faces. The tetrahedron of Gd(III) centres are formed by $\mu_{3}-$ bridging hydroxides. The outermost Gd(III) centres are surrounded by $\mathrm{dmp}^{-}$ligands which show two binding modes: 2.0111 and 3.1112. The Gd(III) ions are eight- or nine-coordinate and the $\mathrm{Gd} \cdots \mathrm{Gd}$ separations in $\mathbf{G d}_{60}$ range from 3.56 to $3.95 \AA$. This compound is clearly a stable thermodynamic product given reaction conditions.
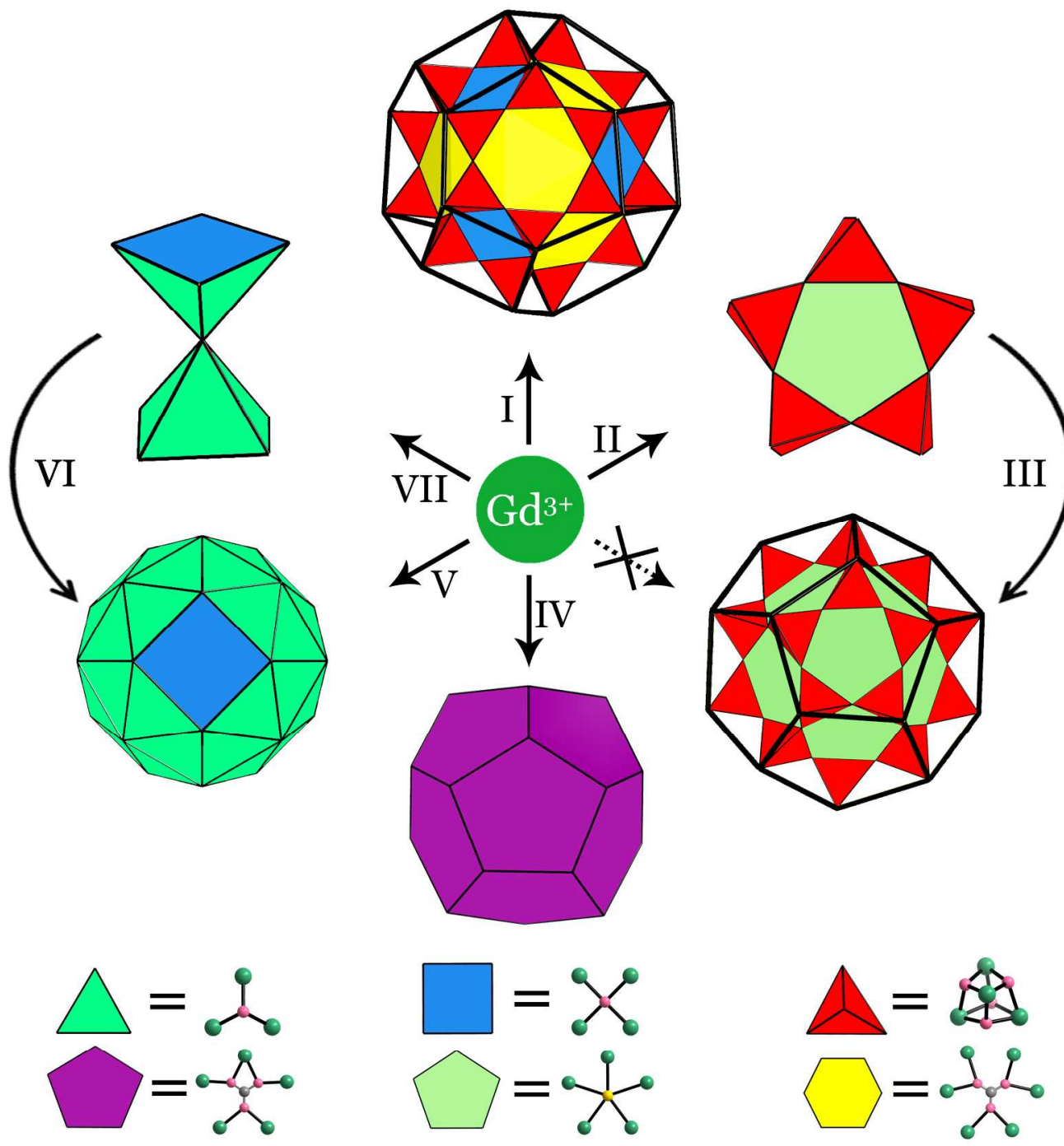

Figure 2. The synthetic strategy of the four high-symmetry spherical Gd(III) polyhedral and two intermediates. Colour codes: Gd, dark green; C, gray; O, pink; Cl, yellow. The synthetic paths from I to VII correspond to the synthesis of $\mathbf{G d}_{\mathbf{6 0}}, \mathbf{G d}_{\mathbf{1 5}}, \mathbf{G d}_{\mathbf{5 0}}, \mathbf{G d}_{\mathbf{2 0}}$, Gd $\mathbf{G}_{\mathbf{3 2}}$, $\mathbf{G d}_{32}, \mathbf{G d}$, respectively (for details see Methods part). The direct synthesis of $\mathrm{Gd}_{50}$ from simple gadolinium salts is not feasible.

To divert the reaction away from $\mathbf{G d}_{60}$ we add sodium chloride as a weak templating agent. Even then, if we heat directly to $130{ }^{\circ} \mathrm{C}$ solvothermally we still produce $\mathbf{G d}_{\mathbf{6 0}}$, however stirring at $90{ }^{\circ} \mathrm{C}$ allows us to trap an intermediate; this compound, $\left[\mathrm{Gd}_{15}\left(\mu_{5}\right.\right.$ $\left.\mathrm{Cl})(\mathrm{OH})_{20}(\mathrm{dmp})_{10}\left(\mathrm{H}_{2} \mathrm{O}\right)_{10}\right]\left(\mathrm{ClO}_{4}\right)_{8} \mathrm{Cl}_{6} \mathbf{G d}_{15}$, contains a $\mu_{5}-$ 
bridging chloride at the centre of a pentagon of $\left\{\mathrm{Gd}_{4}\right\}$ tetrahedron. The $\left\{\mathrm{Gd}_{4}\right\}$ tetrahedron are similar to those in $\mathbf{G d}_{60}$ and it is the presence of a chloride that acts as a template for a pentagon that produces this new cage (Figures 2, 4 and S3). The Hdmp ligands exhibit the 3.1112-mode, chelating the outmost $\mathrm{Gd}(\mathrm{III})$ centres of each $\left\{\mathrm{Gd}_{4}\right\}$ tetrahedron. The Gd(III) ions are coordinated with 7 9 oxygen atoms and the separations between adjacent Gd(III) range from 3.61 to $3.92 \AA$.

The $\mathbf{G d}_{15}$ cage can be isolated and then further reacted under solvothermal conditions $\left(150{ }^{\circ} \mathrm{C}\right)$ to produce $\left.\mathrm{Gd}_{50}\left(\mu_{5}-\mathrm{Cl}\right)_{12}(\mathrm{OH})_{80}(\mathrm{dmp})_{30}\left(\mathrm{H}_{2} \mathrm{O}\right)\right]\left(\mathrm{ClO}_{4}\right)_{20} \mathrm{Cl}_{8} \mathbf{G d}_{50}$ (Figures 2, 3 and S4). The $\mathbf{G d}_{\mathbf{5 0}}$ structure can be described as resulting from twelve $\mathbf{G d}_{\mathbf{1 5}}$ molecules sharing edges of the $\left\{\mathrm{Gd}_{4}\right\}$ tetrahedron. More classically, it is a Keplerate constructed from an outer pentagonal dodecahedron and an encapsulated icosidodecahedron (formed by 12 pentagons and 20 triangles) core. The icosidodecahedron is well known from the $\mathrm{Fe}_{30}$ Keplerate reported by Müller and co-workers, ${ }^{9}$ but the polyhedron in Gd50 containing the outer
Platonic polyhedron is unprecedented. The Gd(III) ions are eight- or nine-coordinate and the $\mathrm{Gd} \cdots \mathrm{Gd}$ separations between the adjacent Gd(III) ions are from 3.61 to $3.93 \AA$. The core is covered by dmp-ligands exhibiting the 3.1112 and 3.0112 fashions.

The chemistry to give $\mathbf{G d}_{\mathbf{1 5}}$ and $\mathbf{G d}_{\mathbf{5 0}}$ relies on chloride templating the formation of pentagons of $\left\{\mathrm{Gd}_{4}\right\}$ tetrahedron. Less predictable pentagons are found when 2-chloro6-hydroxypyridine (Hchp) is reacted with gadolinium nitrate in basic conditions in the $\mathrm{CH}_{3} \mathrm{CN} / \mathrm{CH}_{3} \mathrm{OH}$ mixed solvents; againproducing carbonate as a co-ligand. The result is $\left[\mathrm{Gd}_{20}(\mathrm{chp})_{30}\left(\mathrm{CO}_{3}\right)_{12}\left(\mathrm{NO}_{3}\right)_{6}\left(\mathrm{H}_{2} \mathrm{O}\right)_{6}\right] \mathbf{G d}_{20}$, with the metal sites lying on the vertices of a pentagonal dodecahedron (Figures 2, 3 and S5). Again, this appears to be a new isolated polyhedron for homometallic lanthanide cages; it has been found within $3 \mathrm{~d}-4 \mathrm{f}$ cages, ${ }^{10}$ where the $4 \mathrm{f}$-ions make a dodecahedron inside a icosidodecahedron of $\mathrm{Ni}$ (II) ions. It is also the last of the Platonic solids to be reported as a structural motif in a cage. All of the Gd(III) are ninecoordinate with nearest $\mathrm{Gd} \cdot \cdots \mathrm{Gd}$ separations ranging from 3.82 to $4.00 \AA$. The $\mathrm{Gd}_{20}$ cluster core is encapsulated by the chp-ligands, each adopting a 2.21-coordination mode and with its chlorine atom pointing away from the cluster cage. This pentagonal dodecahedron is far smaller than the same polyhedron found in $\mathbf{G d}_{50}$ (diameter 12.3 cf. $19.5 \AA$, see Figure 4).
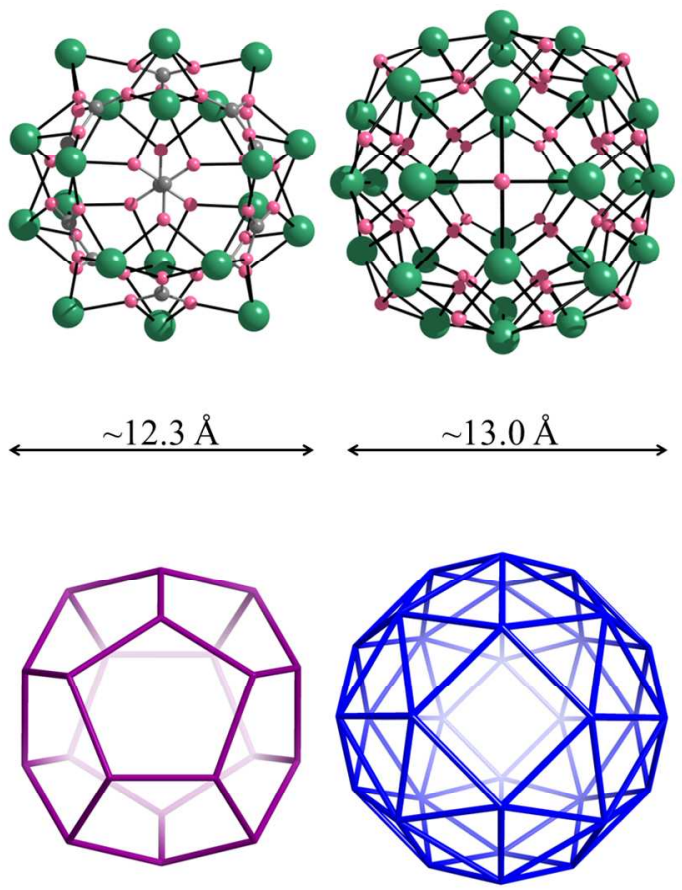

approx. $\mathrm{I}_{\mathrm{h}}, \sim 67 \%$
$\mathrm{O}_{\mathrm{h}}, \sim 81 \%$

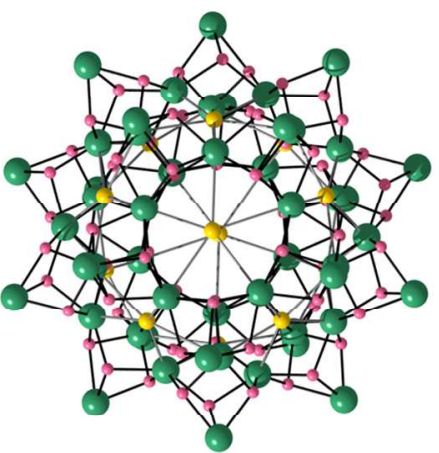

$\sim 19.5 \AA$

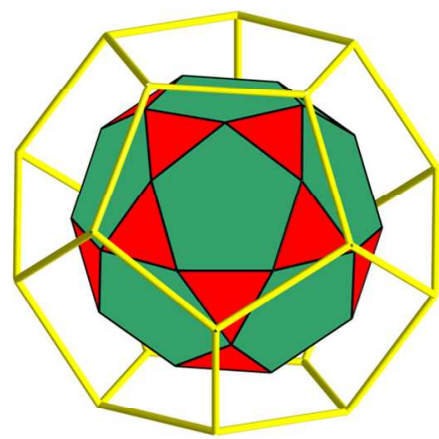

approx. $I_{h}, \sim 67 \%$
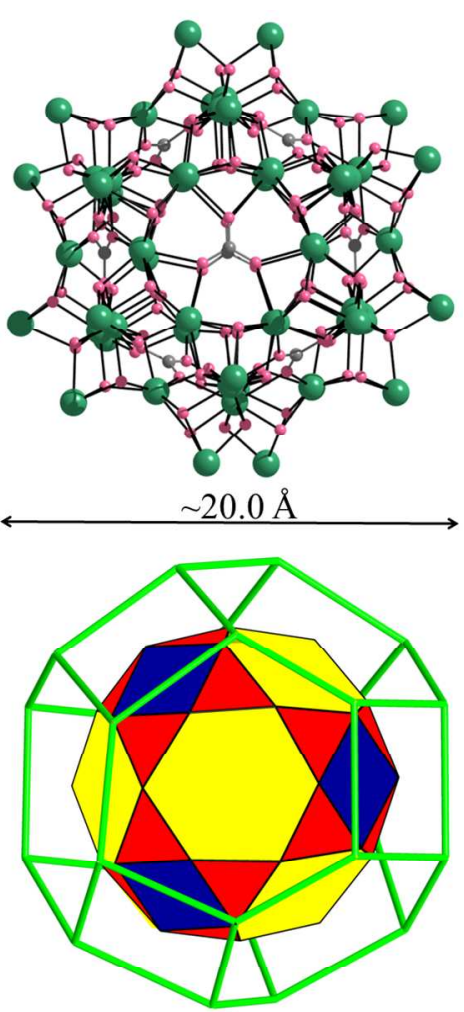

approx. $\mathrm{O}_{\mathrm{h}}, \sim 68 \%$

Figure 3. Structures of four spherical cluster compounds $\mathbf{G d} \mathbf{2 0}, \mathbf{G d} \mathbf{3 2}$, Gd50 and $\mathbf{G d}$ 60, arranged from left to right by increasing nuclearity. Upper, core structures of the four clusters with main bridges and the size (diameter) of each cluster indicated below; lower, topological representation of four clusters, together with the point group and volume ratios ( $\left.V_{\text {polyhedron}} / V_{\text {circumsphere }}\right)$ based on the outmost shell. 
The final polyhedron we discuss is $\left[\mathrm{Gd}_{32}(\mathrm{OH})_{54}(\mathrm{mda})_{12}\left(\mathrm{NO}_{3}\right)_{12}\left(\mathrm{H}_{2} \mathrm{O}\right)_{24} \supset\left(\mathrm{H}_{2} \mathrm{O}\right)_{6}\right](\mathrm{OH})_{6} \quad \mathbf{G d}_{32}$ (mdaH $\mathrm{m}_{2}=\mathrm{N}$-methyldiethanolamine), which is formed when $\mathrm{mdaH}_{2}$ is reacted with gadolinium nitrate at $130{ }^{\circ} \mathrm{C}$ for 3 days. Here we find neither carbonate nor chloride in the structure, and the resulting structure therefore lacks metal faces composed of either hexagons (from carbonate) or pentagons (from chloride or carbonate); instead hydroxides are the major anionic component which leads to formation of linked triangles. The structure features a core of thirty-two Gd(III) sites forming a truncated octahedron of twenty-four Gd atoms with each of its eight regular hexagonal faces capped by a Gd(III) ion; this is the first gigantic gadolinium cluster core with crystallographic $\mathrm{O}_{\mathrm{h}}$ symmetry (Figures 2, 3 and S6). ${ }^{11}$ The nearest Gd $\cdots \mathrm{Gd}$ separations in the core range from 3.60 to $3.87 \AA$. The $\mathrm{Gd}_{32}$ core is surrounded by $3.212 \mathrm{mda}^{2-}$ ligands and monodentate nitrates, providing the eight- or nine-coordinate for all Gd(III) centres.

$\mathbf{G d}_{32}$ is formed after reaction for 3 days; if the reaction time is much shorter (12 hours) we isolate a related smaller cage, Gda cage, $\left[\mathrm{Gd}_{9}(\mathrm{OH})_{10}\right.$ $\left.(\mathrm{mda})_{4}(\mathrm{mdaH})_{2}\left(\mathrm{mdaH}_{2}\right)_{2}\left(\mathrm{NO}_{3}\right)_{7}\left(\mathrm{CH}_{3} \mathrm{OH}\right)_{4}\right]$ (Figures 2, 4 and S7). This is a polyhedron based on two vertex-sharing square-based pyramids; $\mu_{3}$-hydroxides are found at the centre of each triangular face of this polyhedron while the basal distorted square contains a $\mu_{4}$-hydroxides. The Gd(III) ions are eight- or nine-coordinate and the Gd...Gd separations between the adjacent Gd(III) ions are from 3.59 to $3.83 \AA$. It is worth speculating that this structure is the nucleating point for the $\mathbf{G d}_{\mathbf{3 2}}$, i.e. it forms first and then the further hydroxide-centred triangles grow around this cage, companied with the reconstruction of the core structure. Vertex-sharing square pyramidal cores have been reported previously. ${ }^{12}$ In all of the six complexes, the $\mathrm{Gd}-0$ bond lengths locate in the range from 2.23 to $2.82 \AA$, which are normal in Gd(III)-hydroxide clusters.

a

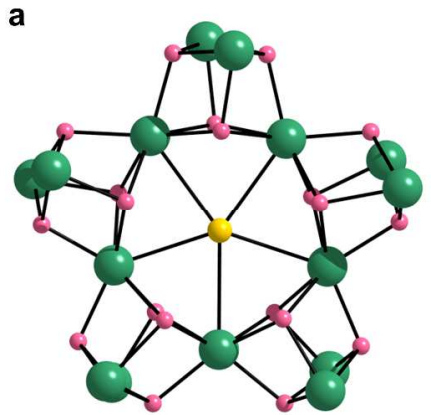

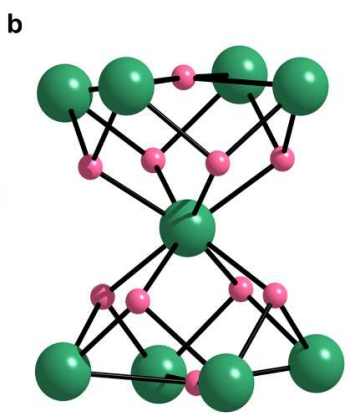

b

Figure 4. The core structures of compounds Gd15 and Gd9.

The synthetic strategy is based on assembling polymetallic fragments which we believe form initially: fragments such as a $\left\{\mathrm{Gd}_{3}\left(\mu_{3}-\mathrm{OH}\right)\right\}$ triangle, $\left\{\operatorname{Ln}_{4}\left(\mu_{4}-\mathrm{OH}\right)\right\}$ square, $\left\{\mathrm{Gd}_{4}\left(\mu_{4}-\mathrm{OH}\right)_{4}\right\}$ tetrahedron (which is built from triangles), $\left\{\mathrm{Gd}_{5}\left(\mu_{5}-\mathrm{Cl}\right)\right\}$ pentagon and $\left\{\mathrm{Gd}_{6}\left(\mu_{6}-\mathrm{CO}_{3}\right)\right\}$ hexagon. Specific anionic templates such as $\mathrm{OH}^{-}, \mathrm{Cl}^{-}$and $\mathrm{CO}_{3}{ }^{2-}$, certain solvents and organic ligands control the distribution of such fragments. This is then followed by aggregation into larger pseudo-spherical clusters. Most of these giant cluster species require a reaction conditions with relatively high temperature and pressure provided in situ by solvothermal conditions; this suggests they are often thermodynamic products. In some cases intermediate structures such as $\mathbf{G d}_{15}$ can be trapped, and the reaction re-directed towards new high symmetry species. The organic ligands involved play an important role in both the hydrolysis of hydrated gadolinium ions and then stabilise the clusters by providing protecting organic shells around the four unprecedented nano-sized spherical clusters. The small inorganic ligands are far more structure directing than the larger polynucleating organic ligands, and this may again appear counter-intuitive but is connected to the flexibility of the organic ligands used here.

In terms of core topology, the four largest cages $\left(\mathbf{G d}_{\mathbf{2 0}}\right.$, $\mathbf{G d}_{\mathbf{3 2}}, \mathbf{G d}_{\mathbf{5 0}}$ and $\mathbf{G d}_{\mathbf{6 0}}$ ) are all closed surfaces with the Euler characteristic $\chi=2$ (Table S2). According to Euler's rule the $\mathbf{G d}_{20}$ core is equivalent to the smallest fullerene structure $\mathrm{C}_{20}$. The $\mathbf{G d}_{\mathbf{3}}$ and the $\mathbf{G d}_{\mathbf{5 0}}$ cores are new in metal cage complexes. ${ }^{11,13}$ Interestingly, the polyhedral surface of the $\mathbf{G d}_{32}$ core is close to an ideal sphere with a ratio of $V_{\text {polyhe- }}$ $\mathrm{dron} / V_{\text {circumsphere }}=\sim 81 \%$, which is higher than an previously reported gadolinium cage (the previous largest one is the $\mathrm{Gd}_{104}$ cage with $\sim 76 \%{ }^{3 \mathrm{~b}}$ ). For comparison, the parent fullerene, $\mathrm{C}_{60}$, has a ratio of $\sim 87 \%$ with twelve pentagons and twenty hexagons.

Magnetic studies. The magnetic properties of six Gd(III) complexes, Gd, $\mathbf{G d}_{\mathbf{1 5}}, \mathbf{G d}_{\mathbf{2 0}}, \mathbf{G d}_{\mathbf{3 2}}, \mathbf{G d}_{\mathbf{5 0}}$ and $\mathbf{G d}_{\mathbf{6 0}}$, were studied on polycrystalline samples. In all cases the high temperature limiting value of the product $\chi T$ (where $\chi$ is the molar magnetic susceptibility) is in good agreement with the calculated spin-only value for the number of independent paramagnetic Gd centres present (Figure 5). For all compounds $\chi T$ decreases with decreasing temperature, indicating the presence of weak antiferromagnetic interactions between the Gd centres but in all cases the value of $\chi T$ is non-zero at $2 \mathrm{~K}$, which is the lowest temperature measured. This indicates paramagnetic states are still populated at this temperature. Temperature-dependent measurements are shown in the supplementary material.

The magnetization $(M)$ of all compounds was measured versus external magnetic field $H$ at low temperatures $(2 \mathrm{~K}$ to $10 \mathrm{~K}$ ) (insert in Figure 5) and is more informative. In each case $M$ saturates at $2 \mathrm{~K}$ at values close to the expected saturation values for the respective number of Gd(III) sites but the increase of $M$ vs. $H$ is by far slower than one would expect for a Brillouin function calculated for $S=7 / 2$ paramagnetic centres. This again supports the evidence for weak antiferromagnetic interactions between the metal centres in all compounds.

Monte Carlo simulations. The size of the metal cages makes it impossible to model the magnetic behaviour using an exact quantum mechanical treatment based on a Heisenberg model approach since the Hilbert space dimension amounts to $(2 S+1)^{N}$, where $S$ is the spin quantum number for Gd and $N$ is the number of Gd centres per cage. However, the cages are suitable for classical spin dynamics and Monte Carlo simulations based on a classical Heisenberg model where the Gd(III) centres are simulated by classical magnetic moments $\vec{m}_{i}$, i.e. three dimensional vectors of length $\left|\vec{m}_{i}\right| \propto \sqrt{S(S+1)}$. ${ }^{14}$ The Hamiltonian used for our simulations is given by 


$$
H=-\frac{1}{2} \sum_{\substack{i, j \\ j \neq i}}^{N} J_{i j} \vec{S}_{i} \vec{S}_{j}-\vec{B} \sum_{i}^{N} \mu_{i} \vec{S}_{i}-\frac{\mu_{0}}{8 \pi} \sum_{i, j}^{N} \frac{\mu_{i}\left(3\left(\vec{S}_{i} \vec{e}_{i j}\right) \vec{e}_{i j}-\vec{S}_{i}\right) \vec{S}_{j}}{r_{i j}^{3}}
$$

Here, the first term contains the Heisenberg exchange interaction between two normalized magnetic moments $\vec{S}_{i}$ and $\vec{S}_{j}$. Therefore, the classical exchange constants $J_{i j}$ have to be scaled with respect to the quantum values $J_{q}$ by

$$
J_{i j}=J_{q} S(S+1)=J_{q} \frac{63}{4}
$$

The second term is the Zeeman term which describes the interaction of each Gd centre with an external field (using the flux density $B$ ). The last term describes the classical dipole-dipole interaction which plays a significant role for very low temperatures in all Gd cages due to the large magnetic moment per ion. The determination of the classical exchange constants $J_{i j}$ has been done by performing classical Monte Carlo simulations and fitting to the experimental $\chi T(T)$ data. In all cases, the contribution from the dipole-dipole interaction was found to be negligible for temperatures $T>2 \mathrm{~K}$. The obtained exchange-coupling constants were then used to calculate the $M(B)$ curves and we find excellent agreement for all temperatures in the field range of $0<B<4 \mathrm{~T}$. The exchange interactions found were: Gd9, $-0.130 \mathrm{~K}, \mathbf{G d}_{20},-0.030 \mathrm{~K}$; Gd $\mathbf{3 2},-0.108 \mathrm{~K}$; Gd50, $-0.127 \mathrm{~K} ; \mathbf{G d}_{\mathbf{6 0}}, J=-0.146 \mathrm{~K}$. For compound $\mathbf{G d}_{\mathbf{1 5}}$ four exchange interactions were needed to fit the data, i.e. $J_{1}=$ $-0.165 \mathrm{~K}, J_{2}=-0.127 \mathrm{~K}, J_{3}=-0.063 \mathrm{~K}, J_{4}=-0.025 \mathrm{~K}$. Here, $J_{1}$ describes the exchange interaction between nearest neighbours with the shortest distance. $J_{2}, J_{3}$ and $J_{4}$ refer to exchange interactions between magnetic centers of larger distances.

The exchange coupling constant of $\mathbf{G d}_{\mathbf{2 0}}$ is significantly smaller when compared to the other systems, because the carbonate is a weak magnetic exchange bridge which was also observed previously in other systems. ${ }^{15}$ For higher fields there is a systematic deviation between experimental and theoretical results at lower temperatures, i.e. the theoretical curves lie above the experimental data. This is an artefact of the classical treatment, i.e. at saturation a classical spin of length $\sqrt{S(S+1)}$ can point completely in the field direction and is therefore larger than the corresponding quantum spin for which the maximum value is given by the quantum number $m_{S}=S$. To the best of our knowledge, this is the first work where a classical Heisenberg model has been successfully used to model the magnetic properties of a rare earth magnetic molecule.
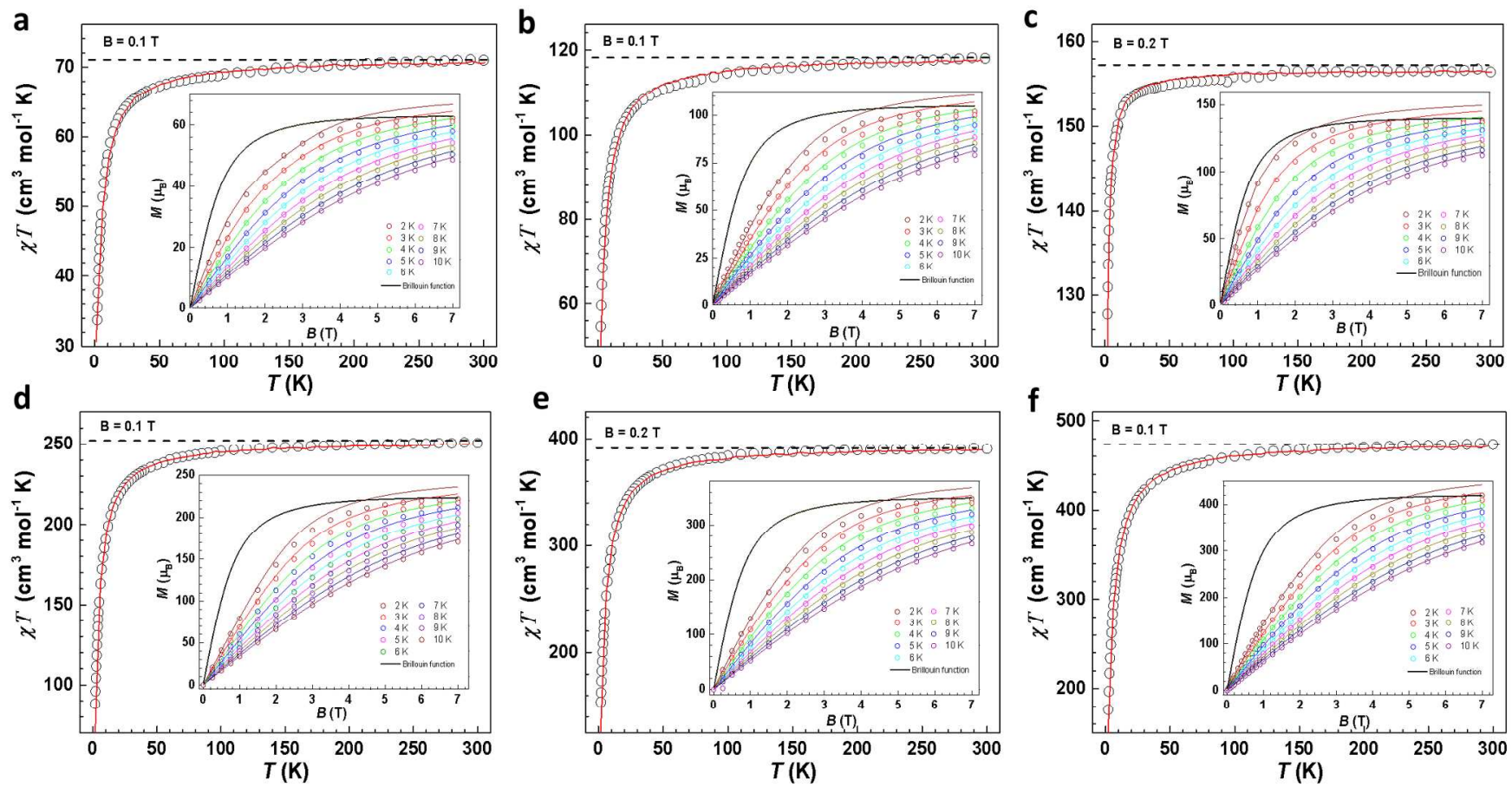

Figure 5. a-d) Plots of $\chi T$ vs. $T$ and isothermal magnetization curves (inserts) and for $\mathbf{G d}_{\mathbf{9}}, \mathbf{G d}_{\mathbf{1 5}}, \mathbf{G d}_{\mathbf{2 0}}, \mathbf{G d}_{\mathbf{3 2}}, \mathbf{G d}_{\mathbf{5 0}}$ and $\mathbf{G d}_{\mathbf{6 0}}, \mathrm{respec}$ tively. Dashed lines are the high-temperature limits for $N$ non-interacting Gd(III) ions with $g=2.0$ in each compounds, respectively. The coloured lines display results from our classical Monte Carlo simulations (see text). The indicated black lines are calculated Brillouin functions for $N$ magnetically isolated Gd(III) ions with $S=7 / 2, g=2.0$ at $T=2 \mathrm{~K}$, respectively.

Pulsed Magnetic Field Studies. To further study the magnetic couplings obtained from Monte Carlo simulations we investigated the magnetization behaviors of these clusters using pulsed magnetic fields up to $10 \mathrm{~T}$ at very low temperatures. At $0.4 \mathrm{~K}$ and under adiabatic condition the magnetization takes a full-cycle sine wave shape for these complexes (Figure 6 and S8). In the down sweep from the highest field the magnetization gradually decreases for all of six complexes, but being higher than that in the first quarter up cycle due to the competition between the thermal relaxation and the fast change of the magnetic field. ${ }^{16}$ To further understand the magnetization behavior the differential magnetizations $(\mathrm{d} M / \mathrm{d} B)$ are plotted against the pulsed field in Figure 6, which give clear magnetization steps (peaks in $\mathrm{d} M / \mathrm{d} B$ vs $B$ plots). For $\mathbf{G d} \mathbf{9}$, these three peaks located at around $0,0.9 \mathrm{~T}$ and $3.0 \mathrm{~T}$, of which the 
near-zero peak is much smaller compared to the others. For $\mathbf{G d}_{15}$, the two peaks are around 0 and 2.0 T. For $\mathbf{G d}_{20}$, one broad peak was observed at low field around zero, indicating the magnetic interactions are almost homogeneous in the system. For $\mathbf{G d}_{\mathbf{3 2}}$, there are two peaks around 0 and 1.0 T. For $\mathbf{G d}_{\mathbf{5 0}}$, there are two peaks located at around 0 and 1.9 T. For $\mathbf{G d}_{60}$, there are three distinct peaks around $0,0.9 \mathrm{~T}$ and $2.5 \mathrm{~T}$.

We reason that these peaks in the derivatives of magnetization indicate inequivalent magnetic superexchangecoupling interactions, for higher fields implying stronger magnetic interactions. Hence, we focus on the magnetostructural correlation and try to elaborate the correlation between these peaks and structural geometries (see below). Magnetic exchange through carbonate and chloride are usually very weak, ${ }^{15}$ and hence the peaks around the zero field in such as $\mathbf{G d}_{\mathbf{1 5}}, \mathbf{G d}_{\mathbf{2 0}}, \mathbf{G} \mathbf{d}_{\mathbf{5 0}}$ and $\mathbf{G d}_{\mathbf{6 0}}$ are presumably caused by bridges of $\mathrm{Cl}^{-}$and $\mathrm{CO}_{3}{ }^{2-}$. The stronger exchange interactions seen are due to $\mathrm{OH}^{-}$or $\mathrm{RO}^{-}$bridges. To classify the Gd...Gd interactions through these groups, we made a survey in literature with reported magnetic exchange-coupling constants () of hydroxide-bridged $\mathrm{Gd}^{\mathrm{III}}$ clusters, many of which comprise similar building units such as triangles, squares, pyramid, octahedron and tetrahedron and show the Gd-O-Gd angles $(\varphi)$ are major structural factors that determine the magnetic interaction. The statistics of these angles and $J$ values are listed in Table S3, from which we can obtain an empirical relationship between them (Figure S9); a linear fitting provides the equation of $J=0.0072 \varphi-0.85245\left(\mathrm{~cm}^{-1}\right)\left(80^{\circ}<\varphi<120^{\circ}\right)$. Based on this expression, $\varphi=118.4^{\circ}$ is critical point that divides ferro or antiferro magnetic interactions.

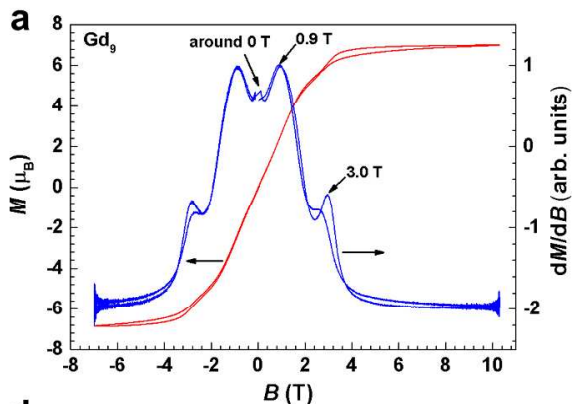

d

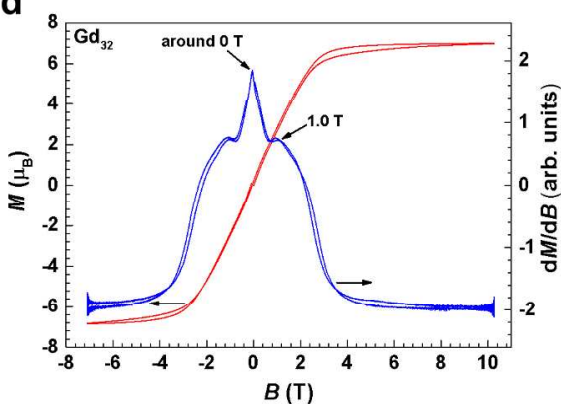

b 8
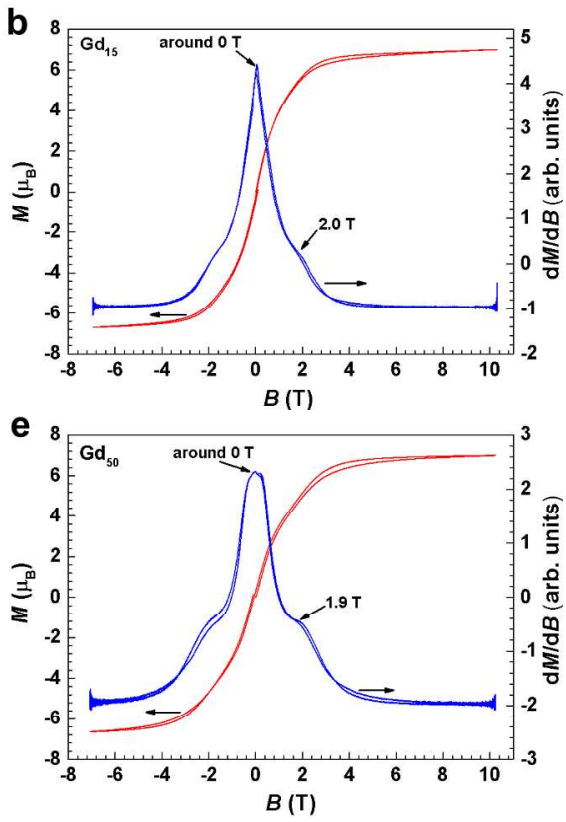

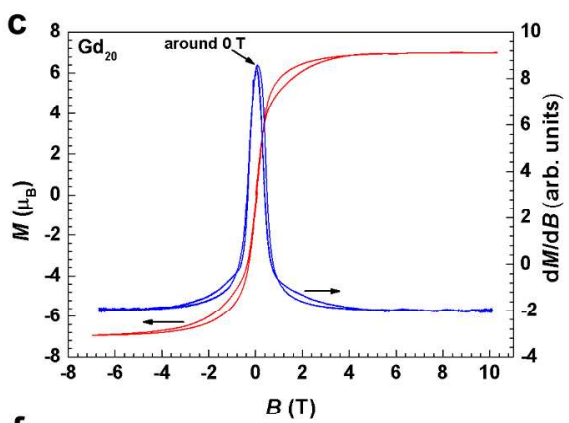

$f \circ$

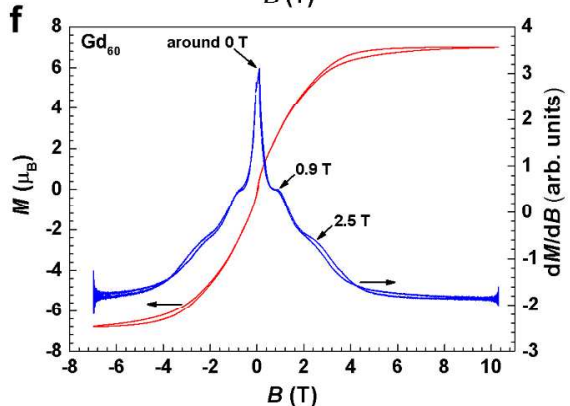

Figure 6. The magnetic hysteresis plots at $0.4 \mathrm{~K}$ normalized by $\mathrm{Gd}(\mathrm{III})$ numbers, together with the field derivatives $(\mathrm{d} M / \mathrm{d} B)$ vs $B$ plots for $\mathbf{G d}_{\mathbf{9}}(\mathrm{a}), \mathbf{G d}_{\mathbf{1 5}}$ (b), $\mathbf{G d}_{\mathbf{2 0}}$ (c), $\mathbf{G d}_{\mathbf{3 2}}$ (d), $\mathbf{G d}_{\mathbf{5 0}}$ (e) and $\mathbf{G d}_{\mathbf{6 0}}$ (f).

For these series of Gd(III) complexes, the magnetic interactions are always antiferromagnetic as $\varphi$ is usually smaller than $120^{\circ}$ for $\mathrm{OH}^{-}$bridges. The correlation shows that the smaller the Gd-O-Gd angle the stronger the antiferromagnetic interactions. By analyzing the Gd-O-Gd angles and the peak positions in the $\mathrm{d} M / \mathrm{d} B$ data we can attribute the following relationships: for $80^{\circ}-96^{\circ}, \mathrm{d} M / \mathrm{d} B$ peaks are around $2.5-3.0 \mathrm{~T}$; for $96^{\circ}-102^{\circ}$, peaks are around $2.0-2.5 \mathrm{~T}$; for $102^{\circ}-108^{\circ}$, peaks are around $1.0-$ $2.0 \mathrm{~T}$; for $108^{\circ}-114^{\circ}$, peaks are around $0-1.0 \mathrm{~T}$ (for detail, see the grouped bond angles in different colors in "Summary of Gd-O-Gd angles and Gd..Gd separations of six Gd(III)-based cages" in Supporting Information). Interestingly, the four "absorption bands" in $\mathbf{G d}_{\mathbf{1 5}}$ coincidently agree with the four magnetic superexchange-coupling constants suggested by the Monte Carlo simulations, though we are unable to correlate them one by one.

\section{CONCLUSION}

To summarize, a series of spherical Gd(III) cages and intermediate species with novel topologies were successfully isolated. $\mathbf{G d}_{\mathbf{2 0}}, \mathbf{G d}_{\mathbf{5 0}}$ and $\mathbf{G d}_{\mathbf{6 0}}$ have approximate cubic or icosahedral symmetry while $\mathbf{G d}_{32}$ has exact $\mathrm{O}_{\mathrm{h}}$ symmetry. The geometry all six Gd(III) cages can be seen as combination of multiple polygons. The magnetism of these cages can be described by a simple classical Heisenberg model, providing the dominant antiferromagnetic interactions. Hence, many intriguing magnetic phenomena arising from geometrical spin-frustration may be expected in these systems. ${ }^{17-24}$ This may pave a new way to obtain new molecular prototypes for the investigation of spin frustration, which will be a solid motivation for both synthetic chemists and theorists. Moreover, from the derivative of the magnetization analyses we can roughly correlate the peak bands and strengths of antiferromagnetic interactions. This may be also viewed as "fingerprints" of certain Gd cages, providing the opportunity to identify molecules under magnetic field.

\section{EXPERIMENT SECTION}


Synthesis. See supporting information.

Materials and Physical Measurements. All reagents are commercially available and were used as received without further purification. Single-crystal X-ray diffraction data were recorded on a Bruker Apex CCD II areadetector diffractometer with graphite-monochromated MoKa radiation $(\lambda=0.71073 \AA$ ) at $100 \mathrm{~K}$. Absorption corrections were applied using multi-scan technique. Their structures were solved by direct method of SHELXTL and refined by full-matrix least-square techniques using the SHELXTL program. Elemental analyses (C, $\mathrm{H}$ and $\mathrm{N}$ ) were performed on a Vario EL III elemental analyzer. Magnetic measurements $(2-300 \mathrm{~K})$ were carried out on powder samples by SQUID magnetometry (Quantum Design MPMS-XL). Diamagnetic corrections were calculated from Pascal constants. The measurements of the magnetization behaviors under the adiabatic condition at $0.4 \mathrm{~K}$ were done by using the pulsed magnetic field, which takes a full cycle.

\section{ASSOCIATED CONTENT}

\section{Supporting Information.}

Crystallographic information files (CIFs) and supporting tables and figures. This material is available free of charge via the Internet at http://pubs.acs.org.

\section{AUTHOR INFORMATION}

\section{Corresponding Author}

zheng.yanzhen@xjtu.edu.cn,

christian.schroeder@fh-bielefeld.de,

richard.winpenny@manchester.ac.uk.

\section{Present Addresses}

$\$$ Department of Chemistry and Environmental Engineering, Anyang Institute of Technology, Henan 455000, China.

\section{Author Contributions}

All authors have given approval to the final version of the manuscript. II These authors contributed equally in this work.

\section{Notes}

The authors declare no competing financial interest.

\section{ACKNOWLEDGMENT}

This work was supported by NSFC (Grant Nos. 21473129 and 21620102002), "National 1000 Young Talents" program, Wuhan National High Magnetic Field Center (2015KF06) and Fundamental Research Funds for the Central University.

\section{REFERENCES}

(1) (a) Kroto, H. W.; Heath, J. R.; O'Brien, S. C.; Curl, R. F.; Smalley, R. E. Nature 1985, 318, 162-163. (b) Haddon R. C. J. Am. Chem. Soc. 1997, 119, 1797-1798. (c) Wang, Y.; Díaz-Tendero, S.; Martín, F.; Alcamí. M. J. Am. Chem. Soc. 2016, 138, 1551-1560.

(2) Richeson, D. Euler's Gem: The Polyhedron Formula and the Birth of Topology, Princeton University Press, 2008.

(3) (a) Echeverría, J.; Casanova, D.; Llunell, M.; Alemany, P.; Alvarez, S. Chem. Commun. 2008, 2717-2725. (b) Peng, J. B.; Kong, X. J.; Zhang, Q. C.; Orendáč, M.; Prokleška, J.; Ren, Y. P.; Long, L. S.; Zheng, Z.; Zheng L. S. J. Am. Chem. Soc. 2014, 136, 17938-17941. (c) Heinl1, S.; Peresypkina, E.; Sutter, J.; Scheer, M. Angew. Chem.
Int. Ed. 2015, 54, 13431-13435. (d) Gao, M.-Y.; Wang, F.; Gu, Z.-G.; Zhang, D.-X.; Zhang, L.; Zhang, J. J. Am. Chem. Soc. 2016, 138, 2556-2559. (e) Wang, Y.; Moses-DeBusk; Stevens, M. L.; Hu, J.; Zavalij, P.; Bowen, K.; Dunlap, B. I.; Glaser, E. R.; Eichhorn. B. J. Am. Chem. Soc. 2017, 139, 619-622.

(4) (a) Müller, A.; Krichemeyer, E.; Bögge, H.; Schmidtmann, M.; Peters, F. Angew. Chem. Int. Ed. 1998, 37, 3359-3363. (b) Müller, A.; Kögerler, P.; Dress, A.W.M. Coord. Chem. Rev. 2001, 222, 193218. (c) Kopilevich, S.; Gil, A.; Garcia-Ratés, M.; Bonet-Ávalos, J.; Bo, C.; Müller, A.; Weinstock. I. A. J. Am. Chem. Soc. 2012, 134, 1308213088. (d) Yin, P.; Wu, B.; Li, T.; Bonnesen, P. V.; Hong, K.; Seifert, S.; Porcar, L.; Do, C.; Keum, J. K. J. Am. Chem. Soc. 2016, 138, 10623-10629.

(5) (a) Schröder, C.; Nojiri, H.; Schnack, J.; Hage, P.; Luban, M.; Kögerler, P. Phys. Rev. Lett. 2005, 94, 017205. (b) Wu, Y.; Krzyaniak, M. D.; Stoddart, J. F.; Wasielewski, M. R. J. Am. Chem. Soc. 2017, 139, 2948-2951.

(6) Schröder, C.; Schmidt, H.-J.; Schnack, J.; Luban, M. Phys. Rev. Lett. 2005, 94, 207203.

(7) Kong, X. J.; Wu, Y.; Long, L. S.; Zheng, L. S.; Zheng, Z. J. Am. Chem. Soc. 2009, 131, 6918-6919.

(8) Harris notation describes the binding mode as $\left[\mathrm{X}_{\mathrm{H}} \mathrm{Y}_{1} \mathrm{Y}_{2} \mathrm{Y}_{3} \ldots \mathrm{Y}_{\mathrm{n}}\right]$, where $\mathrm{X}$ is the overall number of metals bound by the whole ligand, and each value of $Y$ refers to the number of metal atoms attached to the different donor atoms. See Coxall, R. A.; Harris, S. G.; Henderson, D. K.; Parsons, S.; Tasker, P. A.; Winpenny, R. E. P. J. Chem. Soc., Dalton Trans. 2000, 2349-2356.

(9) Müller, A.; Sarkar, S.; Shah, S. Q. N.; Bögge, H.; Schmidtmann, M.; Sarkar, S.; Kögerler, P.; Hauptfleisch, B.; Trautwein, A. X.; Schünemann, V. Angew. Chem. Int. Ed. 1999, 38, 3238-3241.

(10) Kong, X. J.; Ren, Y.-P.; Long, L.-S.; Zheng, Z.; Huang, R.-B.; Zheng, L.-S. J. Am. Chem. Soc. 2007, 129, 7016-7017.

(11) Kong, X. J.; Long, L. S.; Zheng, Z.; Huang, R. B.; Zheng, L. S. Acc. Chem. Res. 2010, 43, 201-209.

(12) Wu, Y. L.; Morton, S.; Kong, X. J.; Nichol, G. S.; Zheng Z. Dalton Trans. 2011, 40, 1041-1046.

(13) Papatriantafyllopoulou, C.; Moushi, E. E.; Christou, G.; Tasiopoulos, A. J. Chem. Soc. Rev. 2016, 45, 1597-1628.

(14) (a) Engelhardt, L.; Schröder, C. Simulating Computationally Complex Magnetic Molecules. Molecular Cluster Magnets, R. E. P. Winpenny, Ed. Singapore: World Scientific, 2011, pp. 245-296. (b) Konstantinidis, N. P. J. Phys.: Condens. Matter 2015, 27, 076001. (c) Konstantinidis, N. P. Phys. Rev. B 2005, 72, 064453. (d) Konstantinidis, N. P. Phys. Rev. B 2007, 76, 104434.

(15) Chen, Y.-C.; Qin, L.; Meng, Z.-S.; Yang, D.-F.; Wu, C.; Fu, Z.-D.; Zheng, Y.-Z.; Liu, J.-L.; Tarasenko, R.; Orendáč, M.; Prokleška, J.; Sechovský, V.; Tong, M.-L. J. Mater. Chem. A 2014, 2, 9851-9858.

(16) Yamase, T; Ishikawa, H.; Abe, H.; Fukaya, K.; Nojiri, H.; Takeuchi, H. Inorg. Chem. 2012, 51, 4606-4619.

(17) Greedan. J. E. Mater. Chem. 2001, 11, 37-53.

(18) Woolfson, R. J.; Timco, G. A.; Chiesa, A.; Vitorica-Yrezabal, I. J.; Tuna, F.; Guidi, T.; Pavarini, E.; Santini, P.; Carretta, S.; Winpenny, R. E. P. Angew. Chem. Int. Ed. 2016, 55, 8856-8859.

(19) Schnack, J.; Schmidt, R.; Richter, J. Phys. Rev. B 2007, 76, 054413.

(20) Schnack, J. C. R. Chemie 2007, 10, 15-20.

(21) Schnack, J. Dalton Trans. 2010, 39, 4677-4686.

(22) Garlea, V. O.; Nagler, S. E.; Zarestky, J. L.; Stassis, C.; Vaknin, D.; Kögerler, P.; McMorrow, D. F.; Niedermayer, C.; Tennant, D. A.; Lake, B.; Qiu, Y.; Exler, M.; Schnack, J.; Luban, M. Phys. Rev. B 2006, 73,024414 .

(23) Sharples, J. W.; Zheng, Y.-Z.; Tuna, F.; McInnes, E. J. L.; Collison, D. Chem. Commun. 2011, 47, 7650-7652.

(24) Sharples, J. W; Collison, D.; McInnes, E. J. L.; Schnack, J.; Palacios, E.; Evangelisti, M. Nat. Commun. 2014, 5, 5321. 
TOC

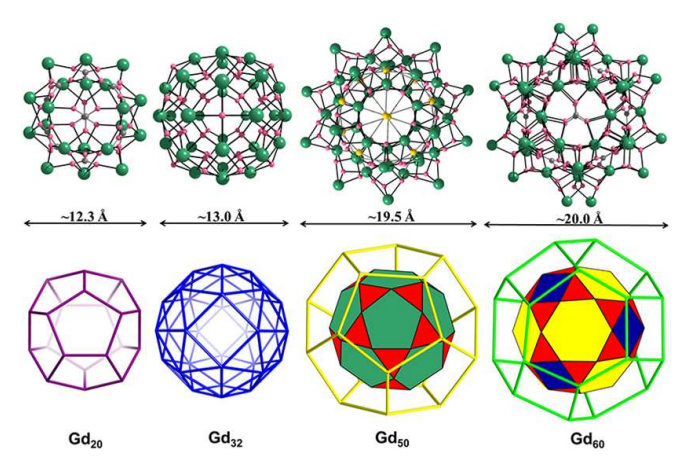

17

18

19

20

21

22

23

24

25

26

27

28

29

30

31

32

33

34

35

36

37

38

39

40

41

42

43

44

45

46

47

48

49

50

51

52

53

54

55

56

57

58

59

60 